\title{
Stromal Senp1 promotes mouse early folliculogenesis by regulating BMP4 expression
}

\author{
Shu Tan ${ }^{1 \dagger}$, Boya Feng ${ }^{2 \dagger}$, Mingzhu Yin ${ }^{1}$, Huanjiao Jenny Zhou' ${ }^{1}$, Ge Lou ${ }^{3}$, Weidong Ji, Yonghao Li ${ }^{4}$ \\ and Wang $\operatorname{Min}^{1,2^{*}}$
}

\begin{abstract}
Background: Mammalian folliculogenesis, maturation of the ovarian follicles, require both growth factors derived from oocyte and surrounding cells, including stromal cells. However, the mechanism by which stromal cells and derived factors regulate oocyte development remains unclear.

Results: We observed that SENP1, a small ubiquitin-related modifier (SUMO)-specific isopeptidase, was expressed in sm22a-positive stromal cells of mouse ovary. The sm22a-positive stromal cells tightly associated with follicle maturation. By using the sm22a-specific Cre system, we show that mice with a stromal cell-specific deletion of SENP1 exhibit attenuated stroma-follicle association, delayed oocyte growth and follicle maturation with reduced follicle number and size at early oocyte development, leading to premature ovarian failure at late stages of ovulating life. Mechanistic studies suggest that stromal SENP1 deficiency induces down-regulation of BMP4 in stromal cells concomitant with decreased expression of BMP4 receptor BMPR1b and BMPR2 on oocytes.
\end{abstract}

Conclusions: Our data support that protein SUMOylation-regulating enzyme SENP1 plays a critical role in early ovarian follicle development by regulating gene expression of BMP4 in stroma and stroma-oocyte communication.

\section{Background}

Folliculogenesis is the maturation of the ovarian follicle, a densely packed shell of somatic cells that contains an immature oocyte. Mouse ovarian development can be divided into several steps: (1) germ cysts breakdown and primordial follicle formation, (2) primordial follicle activation and development to advanced-stage follicles, (3) ovulation or apoptosis. Within the ovary in mice, primordial germ cells arrested in urogenital ridges to undergo mitosis, results in oocytes cluster, which subsequently to form germ cell clusters [1-6]. Following the programmed breakdown of germ cell cysts shortly after birth, only one-third of individual oocytes enveloped by a layer of flat somatic pregranulosa cells, which eventually become primordial follicles [7-9]. With continuous loss and apoptosis of oocytes after birth by unknown

\footnotetext{
*Correspondence: wang.min@yale.edu; 1256830791@qq.com

${ }^{\dagger}$ Shu Tan and Boya Feng contributed equally to this work

${ }^{2}$ Center for Translational Medicine, The First Affiliated Hospital, Sun Yatsen University, Guangzhou 510080, China

Full list of author information is available at the end of the article
}

mechanisms, selected primordial follicles recruit a single layer of cuboidal granulosa cells with oocytes grow inside to form primary follicles, which in turn mature into advanced follicles. It is known now that the oocytes numbers in adult are tightly associated with the finite primordial follicle reservoir. Moreover, the breakdown of germ cell clusters, the cell proliferation in primordial follicle formation and the transition from primordial follicle into primary follicle is critical for subsequent folliculogenesis, i.e., progression of a number of small primordial follicles into large preovulatory follicles [7-9]. Recent studies suggest that folliculogenesis requires both oocyte intrinsic self-organization and complex communications with surrounding somatic cells, involving multiple autocrine and paracrine signaling pathways [10-14]. In particular, specific cytokines and growth factors derived from stromal cells are required for activation of primordial follicle and maturation of oocytes $[7-9,15]$.

The small ubiquitin-like modifier (SUMO) can be covalently attached to a large number of proteins through formation of isopeptide bonds with specific lysine 
residues of target proteins [16]. SUMO (SUMO1, 2 and 3) with SUMO1 more broad specificity [17], is covalently attached to substrate proteins via an isopeptide bond between a C-terminal glycine and a lysine residue in the substrate. A consensus SUMO acceptor site has been identified consisting of the sequence $\varnothing \mathrm{KXE}(\varnothing$ is a large hydrophobic amino acid and $\mathrm{K}$ is the site of SUMO conjugation). The consequence of SUMOylation on protein function is substrate specific, regulating protein stabilization, localization, protein-protein or protein-DNA interactions, and/or biochemical activities. SUMOylation is a dynamic process that is mediated by activating (E1), conjugating (E2), and ligating (E3) enzymes and is readily reversed by a family of SUMO-specific proteases with 6 members [18]. SENP1 is a protease that appears to be localized in several compartments and deconjugates a large number of SUMOylated proteins [18-20]. Recently, protein post-translational modification SUMOylation has been reported to play an important role in germ cell function, especially in mammalian meiosis [21-23]. Several studies have characterized expression of SUMO-1 and SUMO-2/3 in oocytes. While SUMO$2 / 3$ proteins are localized in nucleoplasm, SUMO- 1 is concentrated at spindle organization and chromosome in transcriptionally active oocytes with little location on nuclear membrane in quiescent oocytes. Moreover, this specific localization of SUMO-1 plays a critical role during oocytes maturation [21-23]. It has also been reported that differential localization of SENP1 regulate SUMOylation in a temporal and spatial fashion along the oocyte meiosis procession $[24,25]$. However, little is known about role of protein SUMOylation in stromal cells surrounding oocyte in regulating follicle development and oocyte maturation is unclear. Here, we show that stromal deletion of SENP1 in mice, by increasing cellular SUMOylation and decreasing BMP4 expression, retards oocytes growth and follicle formation at early developmental stage.

\section{Results \\ SM22a-positive stromal cells surround germ cells and oocytes}

Classical studies identified that stromal/somatic cells interact closely with germ cells as early as $14.5 \mathrm{dpc}$ (day past coitum) in mouse ovarian development [1-3]. Stromal cells and stromal progenitor cell insert between closely associated germ cells followed by germ cysts breakdown as part of the progress results in primordial follicle formation. Moreover, these stromal progenitor cells undergo differentiation and maturation into various cell types, including flat epithelial cells, cuboidal granulosa cells and theca cells that support oocyte maturation (Fig. 1a). It is reported that the stromal cells secret growth factors critical for oocyte maturation. However, the nature and function of these stromal cells have not been investigated. By performing co-immunofluorescence staining with germ cell marker VASA protein (also named DDX4/MVH), we initially observed that stromal cells express smooth muscle cell progenitor marker SM22 $\alpha$ (Fig. 1b, c). VASA expression could be detected as early as primordial germ cells (PGCs) and was expressed throughout oocyte development stages, including primary, secondary and mature oocytes. $\mathrm{VASA}^{+}$primordial and primary follicles were juxtaposed with SM22 $\alpha^{+}$ cells at D0 (Fig. 1b), and subsequently the mature oocytes along with granulosa cells were tightly surrounded by SM22 $\alpha^{+}$stromal cells (Fig. 1c for D7).

\section{Stromal SENP1 deletion accelerates premature ovarian failure at late stages of ovulating life}

Based on the role of protein SUMOylation in germ cell function, we reasoned that a specific deletion of deSUMOylase SENP1 in SM22 $\alpha$-positive stromal cells would affect follicle formation. To test our hypothesis, we created mice with an SM22 $\alpha$-specific SENP1 deletion by mating SENP1 ${ }^{\mathrm{f} / \mathrm{fl}}$ with SM22 $\alpha$-Cre mice (Fig. 2a). We examined the SM22 $\alpha$ lineage in the ovary by mating SM22 $\alpha$-Cre with $\mathrm{mG} / \mathrm{mT}$ reporter mice [26], and results indicated that SM22 $\alpha$ cells were located within ovarian stroma (Fig. 2b). SENP1 deletion in SM22 $\alpha$-positive cells was verified by qRT-PCR and Western blotting by isolating SM22 $\alpha$-positive cells from ovaries of WT $\left(\mathrm{SENP}^{\mathrm{f} / \mathrm{fl}}\right)$ and SENP1-smKO (SM22 $\alpha$-Cre; SENP1 $\left.{ }^{\mathrm{f} / \mathrm{fl}}\right)($ Fig. 2c, d). To explore how protein SUMOylation affects the reproductive life in mouse, ovaries sections from WT and SENP1smKO mice at various ages (3-9 weeks) were examined by $H \& E$ staining and the numbers of follicles at various stages were quantified (Fig. 2e). The number of follicles in ovaries from SENP1-smKO mice was significantly reduced compared to WT mice at all ages ( 3 and 6 weeks till 8 months), with more severe reduction at older ages (>8 month older) (Fig. 2e, f). At 8 months postpartum, different stages developing follicles (primary, secondary and advanced stages) and corpus lutuem were detected in WT ovaries. SENP1-smKO ovaries contained very few follicles and much more empty follicles without oocytes, implicating that ovaries were degenerated.

\section{Stromal SENP1 deletion attenuates oocyte growth and follicle formation}

The premature ovarian failure of SENP1-smKO mice at 8 months old prompted us to examine early stages of ovarian development, including activation of primordial, primary and secondary follicles. Germ cell cysts were defined as two or more oocytes that were not individually separated by stromal cells. Primordial follicles 


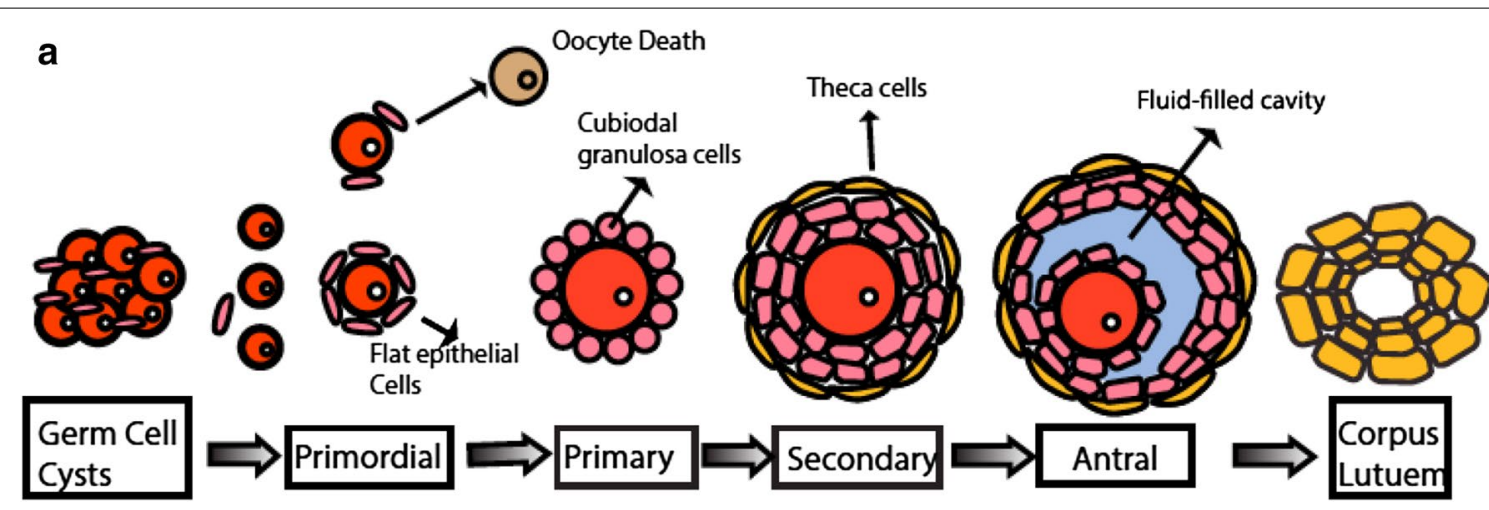

b
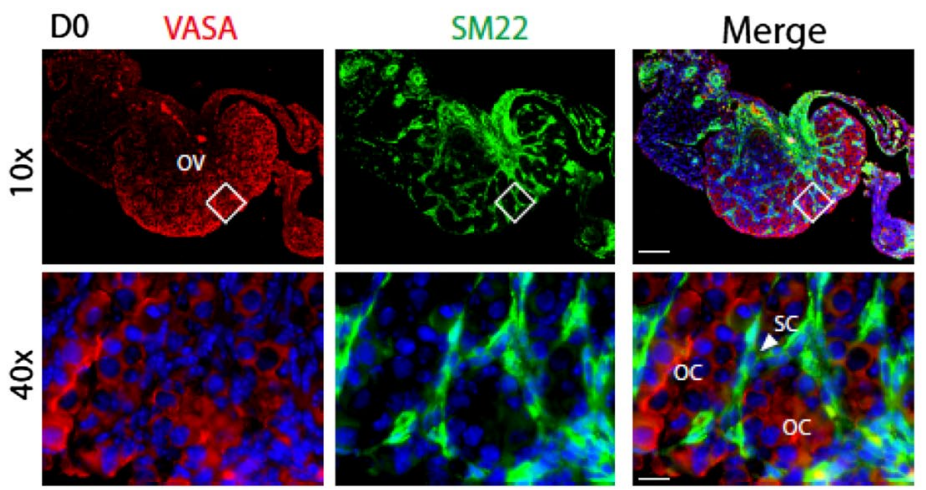

C
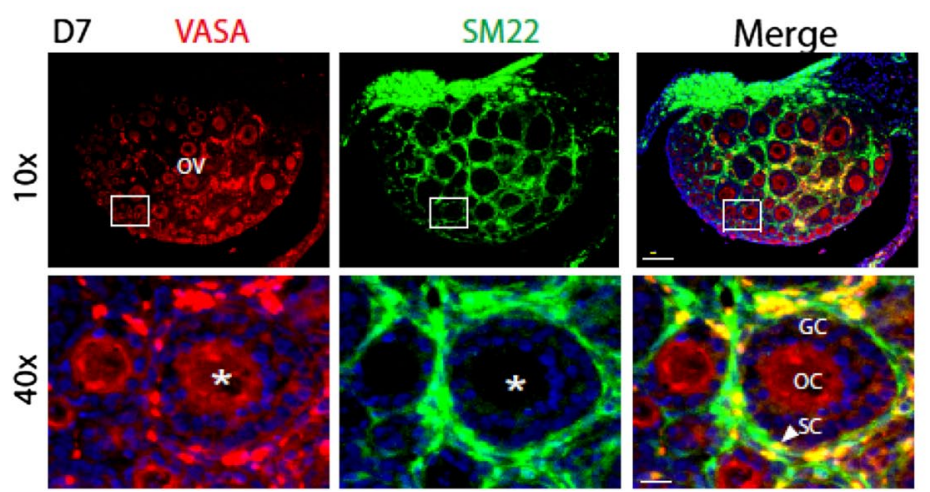

Fig. 1 Identification of SM22a-positive stromal cells in mouse ovary development. a Diagram for mammalian oocyte development within ovarian follicles. Shortly after birth, mouse germ cell cysts undergo programmed breakdown while single oocyte survive to form primordial follicles. As follicles grow, granulosa cells keep proliferating (one-layer to multi-layer) until fluid-filled cavities (the antrum) appear between the layers of somatic cells at later stage of follicle development. At the same time, stromal cells (not shown in the diagram) develop into mature stage to surround the layers of theca/granulosa cells. b, c SM22a stromal cells surround oocytes. DDX4 (DEAD-box helicase 4; also known as VASA) and stromal cell marker SM22a were used co-immunofluorescence in ovaries of newborn (D0; b) and 7-day-old (c; D7) WT mice. In newborn ovaries, stromal cells (green) were arranged in strips within the interval of germ cells (red). Stromal cells become mature and envelope layers of granulosa cells in advanced follicles. Scale bars $60 \mu \mathrm{m}$ in $\times 10$ images; $15 \mu \mathrm{m}$ in $\times 40$ images. OV ovary, OC oocyte, GC granulosa cell, SC stromal cell. Asterisks indicate the same oocyte in the merged panel

were defined as small oocytes $(<20 \mu \mathrm{m})$ surrounded by flat epithelial cells; primary follicles were defined as having larger oocytes $(>20 \mu \mathrm{m})$ surrounded by a single layer of cuboidal and proliferative granulosa cells; secondary follicles were defined as larger oocytes surrounded by two or more layers of granulosa cells (see Fig. 1a) [1-3]. On basis of previous studies in mouse ovarian development, primary-stage follicles and secondarystage follicles are formed on postnatal day 3-7 [1-6]. We examined oocytes growth and follicle development 
a

Exon4

Exon5

Exon6

Exon7

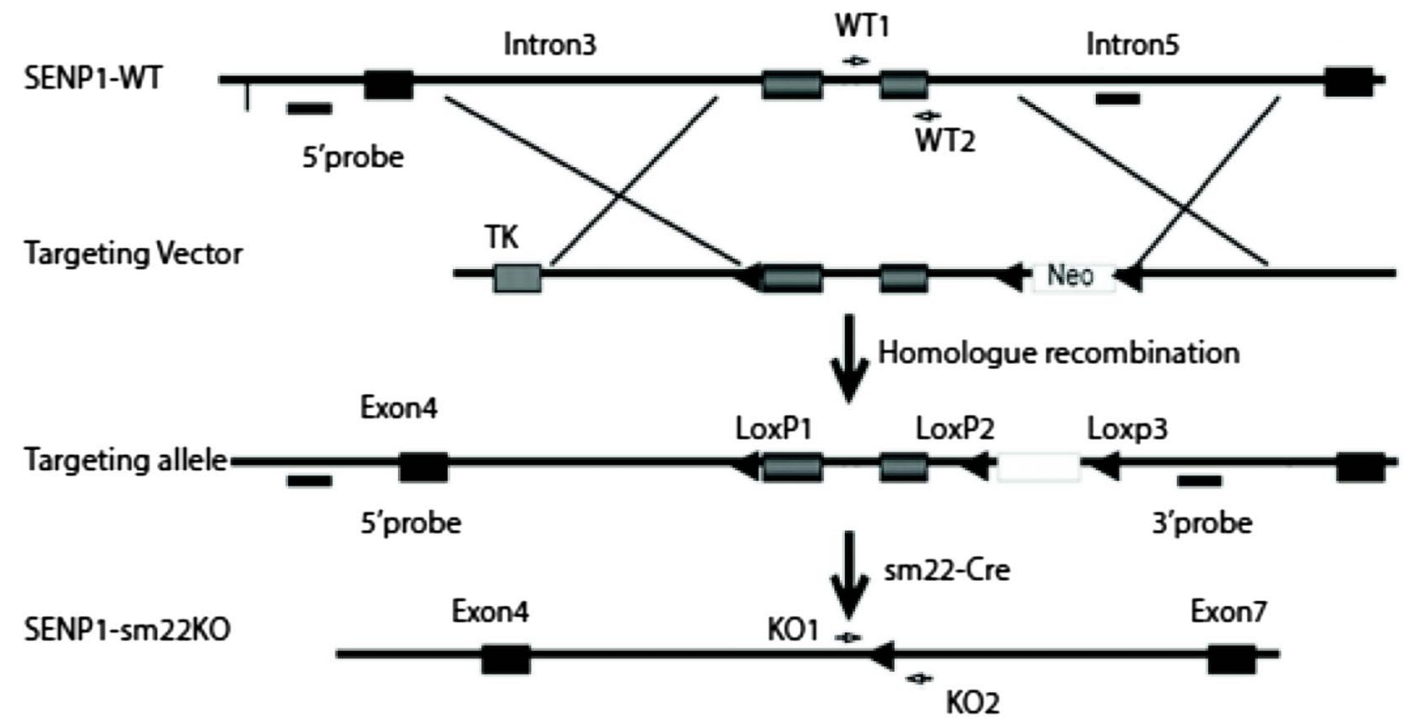

b
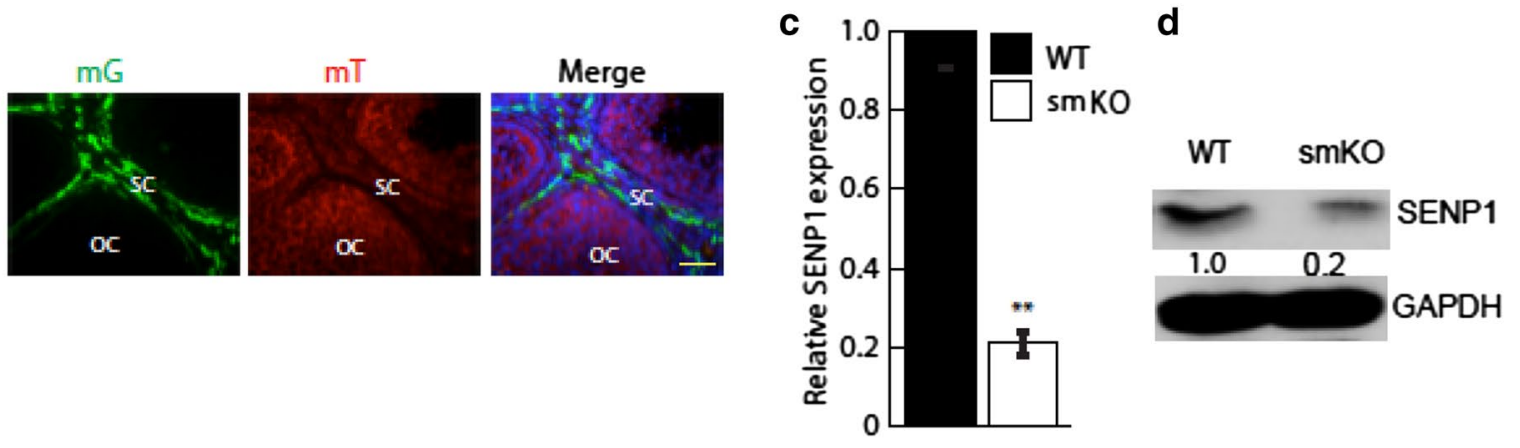

d

e



f
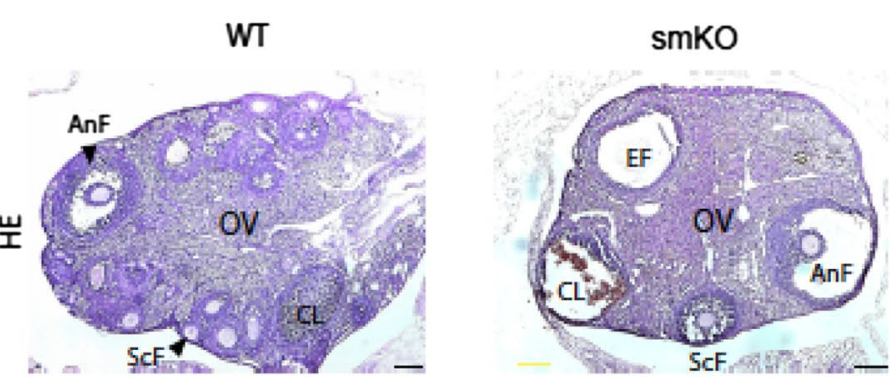

Fig. 2 Premature ovarian failure appeared in later stage of SENP1-smKO mice ovulating life. a Stromal deletion of SENP1 with SM22a-Cre. SENP1 lox/ lox mice were generated based on homologous recombination. SENP1 lox/lox mice were mated with SM22a-Cre to obtain stromal-specific SENP1deficient (SENP1-smKO) mice. $5^{\prime}$ and 3' probes were used for Southern blot. Primers WT1/2 and KO1/2 were used for genotyping. b SM22a cells

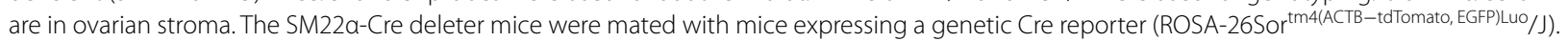
Stromal cells show GFP-positive, suggesting these cells are SM22a lineage. c, d SENP1 deletion in SM22a stromal cells. SM22a-positive stromal cells were isolated from ovaries of SENP1 1ox/lox (WT) and SENP1-smKO female mice. SENP1 expression was detected by qRT-PCR (b) and Western blotting (c). Normalized mRNA and protein levels (with GADPH) are presented as fold changes by taking WT group as 1.0. Data are shown as mean \pm SEM, $\mathrm{n}=3$, female. e Stromal SENP1 deletion reduces follicle numbers. Number of total follicle in ovaries of WT and SENP1-smKO mice at ages of 3, 6 weeks and 8 months. Data are presented as mean $\pm S E M, n=5,{ }^{*} P<0.05,{ }^{* *} P<0.01$. f Premature ovarian failure in aged SENP1-smKO mice. Representative Hematoxylin and Eosin staining of ovaries from 9-month-old WT and SENP1-smKO $(n=5)$. Scale bars $160 \mu m$. OV ovary, SCF secondary follicle, AnF antral follicle, CL corpus lutuem 
in WT and SENP1-smKO pups on day 3-7 by H\&E staining and VASA immunostaining. H\&E staining suggested that oocytes in WT were evenly distributed through ovary with uniform sizes on day 3 and day 7 . Oocytes in SENP1-smKO ovaries were much smaller with variable sizes on day 3 and even on day 7 (Fig. 3a, b with quantifications in Fig. 3c). In contrast, mice with a SENP1 deletion in vascular endothelial cells (SENP1ecKO) did not significantly alter ovarian development and oocyte maturation compared to WT ovaries (Additional file 1: Figure $\mathrm{S} 1 \mathrm{~A}-\mathrm{C}$ ). These data suggest a specific role for SENP1 in SM22 $\alpha$-positive stromal cells.

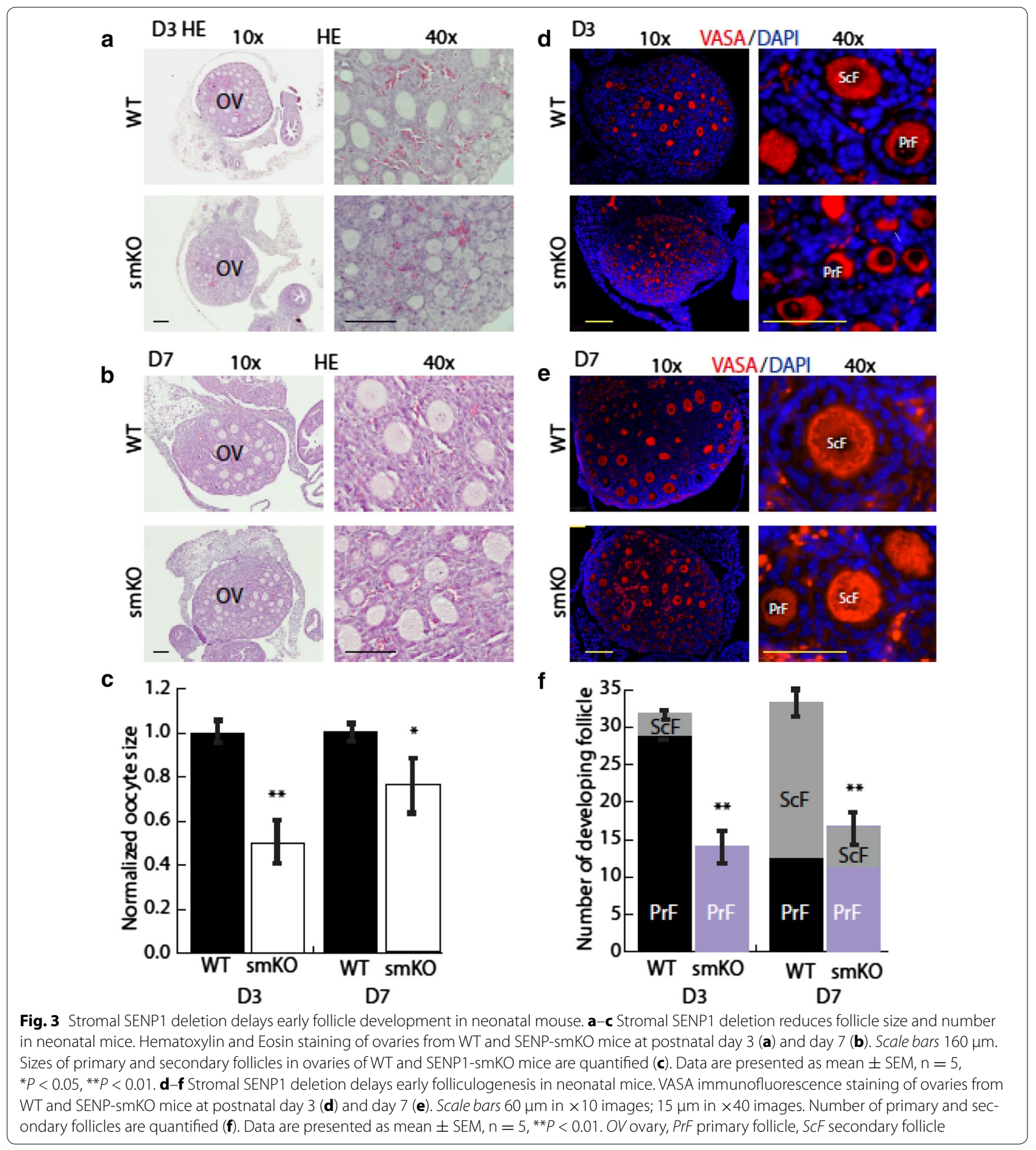


SENP1 ASA staining indicated that WT ovaries on postnatal day 3 contained a majority of primary follicles which rapidly matured into secondary follicles on day 7. However, SENP1-smKO oocytes were smaller sizes with various stages of oocytes, including germ cell cysts (GCC), primordial follicles (PrmdF), primary follicles (PrF) and secondary follicles (ScF) (Fig. 3d). By postnatal day 7, WT oocytes were at a stage of secondary follicle but most ovaries of SENP1-smKO mice were still at an early stage of primary follicle with smaller sizes (Fig. 3e). The number of developing follicles (both primary follicle and secondary follicles) was significantly lower in the SENP1-smKOs on both D3 and D7 (Fig. 3f). These results suggest that SENP1-smKO mice exhibit delayed oocyte growth and follicle development from the primordial follicle activation to advanced stages. We cannot exclude the possibility that folliculogenesis in SENP1-smKO mice might be arrested at even earlier stage, Cyst or primordial stage, or drop out of the developmental process at very early stage.

\section{SM22a stromal cells are disorganized with altered expression of BMPs in ovaries of SENP1-smKO mouse}

To further detect changes of stromal cells in SENP1smKO mice in which a delayed oocytes growth and decreased follicles formation were observed in early ovarian development, ovaries from WT and SENP1smKO mice stained with anti-SM22 $\alpha$ antibody, we detected well-distributed SM22 $\alpha$ expression in stromal cells surrounding the follicles of WT ovaries. In contrast, un-uniform distributions of SM22 $\alpha$ stromal cells were observed in SENP1-smKO ovaries at both D0 and D3 (Fig. 4a). SENP1 deletion in SM22 $\alpha^{+}$cell was confirmed by co-immunostaining with anti-SENP1 and antiSM22 $\alpha$ (Fig. 4b). BMPs have been identified important roles during formation, growth and maturation of ovarian follicles in mammals [27-32]. Among them, BMP4 derived from stromal/somatic cells is critical in promoting the survival and development of primordial follicles in the neonatal ovary, especially at the primordial-toprimary follicle transition. To investigate if SENP1 regulates gene expression of stromal BMPs, we carried out a series of experiments using the primary human ovarian stromal cells (from Department of Obstetrics and Gynecology, Yale School of Medicine), which proved to be SM22 $\alpha$ positive (Fig. 4c). We determined effects of SENP1 knockdown on BMPs expression in stromal cells by qRT-PCR. BMP4, but not other BMPs, was significantly reduced by SENP1 siRNA silencing (Fig. 4d). Expression of BMP4 protein level was also significantly decreased in SENP1-knockdown stromal cells (Fig. 4e). These results suggest that SENP1 is critical for BMP4 expression in stromal cells.

\section{Stromal SENP1 deletion reduces expression of BMP4 receptor BMPR1B and BMPR2 on oocytes}

To further investigate potential involvement and relative functions of stromal SENP1 in regulating early stage of oocytes and follicles development, we further examined expressions of BMP4 and its cognate receptors BMPR1A, $B M P R 1 B$ and BMPR2 in vivo. Consistent with previous reports ref, BMPR1B and BMPR2, but not BMPR1A, were highly expressed in developing ovaries (Fig. 5a). To determine which cell type contributes to BMPR expression, we performed co-immunostaining for BMP4 and its receptors. BMP4 was primarily expressed by SM22 $\alpha$ stromal cells surrounding follicles in WT ovaries. Consistent with the in vitro findings, BMP4 was greatly reduced in the stromal cells of SENP1-smKO ovaries (Fig. 5b). As previously reported, BMPR1B and BMPR2 were detected on oocytes (Fig. 5c-e). Interestingly, we observed reduced expression of BMPR1B (Fig. 5c, d) and BMPR2 (Fig. 5e) in oocytes of SENP1smKO mice, consistent with a delayed maturation of oocytes in these mice.

\section{Discussion}

Folliculogenesis is a complex process that depends on numerous factors including both extra-ovarian and intraovarian factors. It has long been recognized that oogenesis and folliculogenesis require complex bidirectional signaling between the oocyte and the surrounding stro$\mathrm{mal} / \mathrm{somatic}$ cells; while stromal cells support oocyte development, oocytes promote surrounding stromal cells differentiation and proliferation [10-14]. However, the molecular mechanism underlying the interactions between oocyte and stroma remains unknown. Our study supports that SENP1 in ovarian stroma is crucial for maintenance and survival of folliculogenesis. It is evident that mice with a deletion of the SENP1 gene in $\mathrm{SM}_{2}{ }^{+}$ovarian stromal cells (SNEP1-smKO) exhibit an incomplete breakdown of germ cell cysts with reduced number of primary and secondary follicles on both postnatal day 3 and day 7 . Consequently, the number of total follicles in adolescent and adulthood of SENP1-smKO mice maintain fewer than wild-type littermates, leading to premature ovarian failure in old ( $>8$ months of age) SENP1-smKO mice. In humans, premature ovarian failure, also known as premature ovarian insufficiency (POI) or primary ovarian insufficiency, is the loss of function of the ovaries before age 40 [10]. Our SENP1-smKO mice may provide a useful mouse model for human POI to investigate the pathogenesis and underlying mechanism for POI. The distribution of SM22 ${ }^{+}$ovarian stromal cells surrounding the follicles in SENP1-smKO mice was drastically disrupted at early stage, suggesting that alterations of cellular organization of follicle contribute to defective development process. 


\section{a SM22/DAPI}

D0
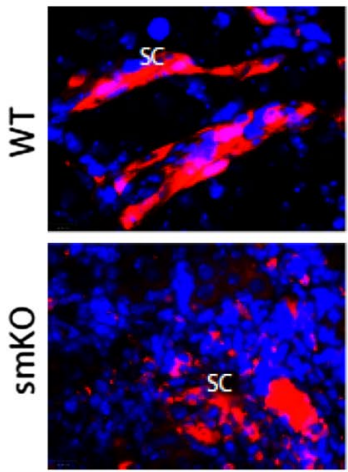

b
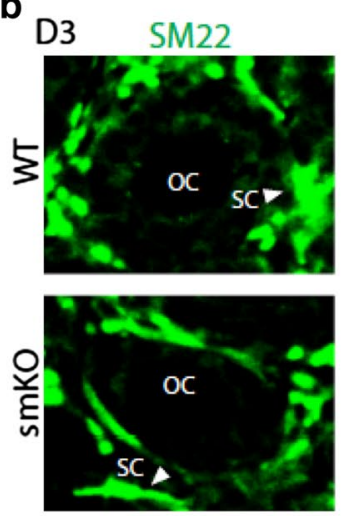

C Human ovarian stromal cell

SM22

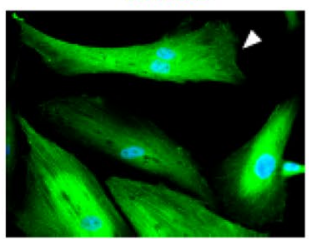

d

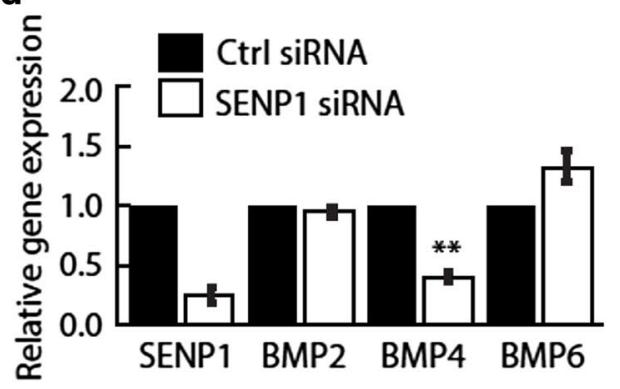

D3
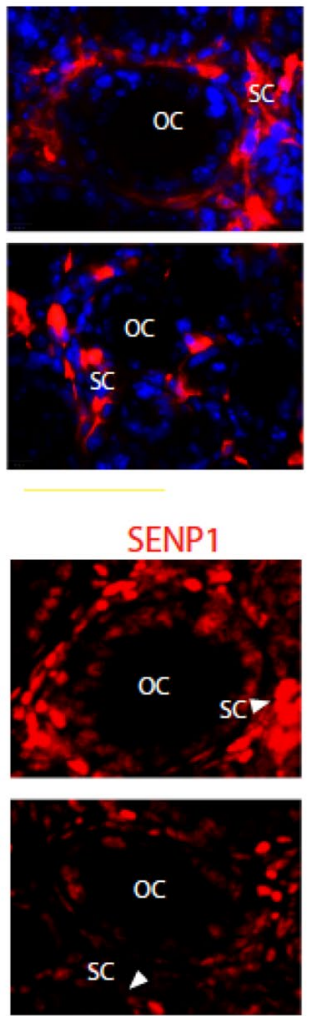

Phalloidin

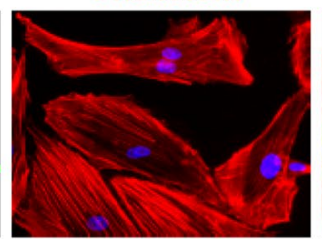

e
D7
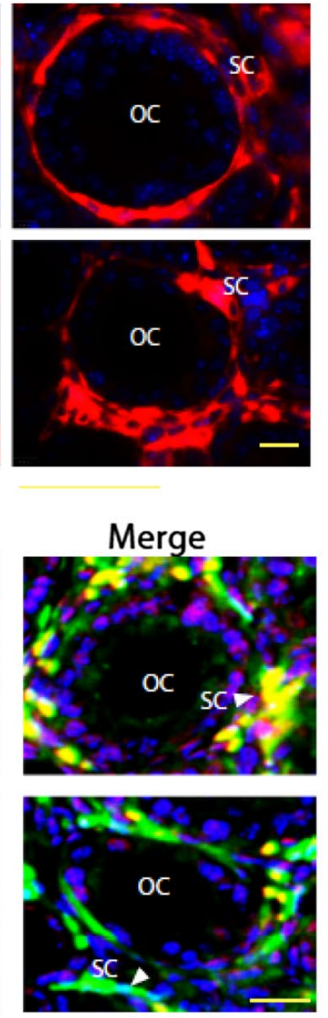

Merge
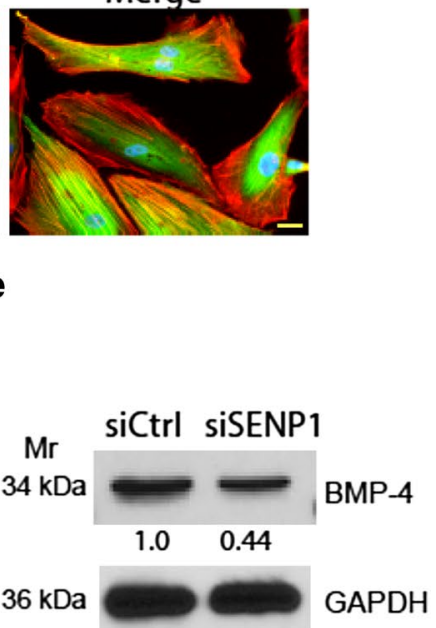

Fig. 4 SENP1-deficient stromal cells are disorganized with reduced expression of BMP4. a SENP1-deficient stromal cells are disorganized. Stromal cell marker SM22a immunofluorescence in ovaries of WT and SENP1-smKO mice at D0, D3 and D7. SM22 $\mathrm{a}^{+}$stromal cells gradually mature to tightly wrap advanced follicles In WT ovaries. However, SENP1-smKO stromal cells exhibit delayed maturation and loosely surround follicles. Scale bars $15 \mu \mathrm{m}$. OC oocyte, SC stromal cell. b SENP1 and SM22a co-immunofluorescence staining of ovaries from WT and SENP-smKO mice. c Immunofluorescence staining of sm22a and phalloidin of human primary ovarian stromal cells. Scale bars $10 \mu \mathrm{m}$. d, e SM22a ${ }^{+}$stromal cells were transfected with control siRNA or SENP1 siRNA. d SENP1, BMP2, BMP4 and BMP6 mRNAs were determined by qRT-PCR. Normalized mRNA levels (with GADPH) are presented as fold changes by taking control siRNA group as 1.0. e Protein level of BMP4 was determined by western blot with respective antibodies. Normalized protein levels (with GADPH) are presented as fold changes by taking control siRNA group as 1.0. Three independent experiments were performed 


\section{a WT smKO \\ $60 \mathrm{kDa} \quad$ BMPR1A \\ $57 \mathrm{kDa}=\mathrm{BMPR} 1 \mathrm{~B}$ \\ $\begin{array}{ll}1.0 & 0.3\end{array}$ \\ $115 \mathrm{kDa}$ BMPR2 \\ $34 \mathrm{kDa}$

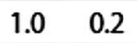 GAPDH}

b
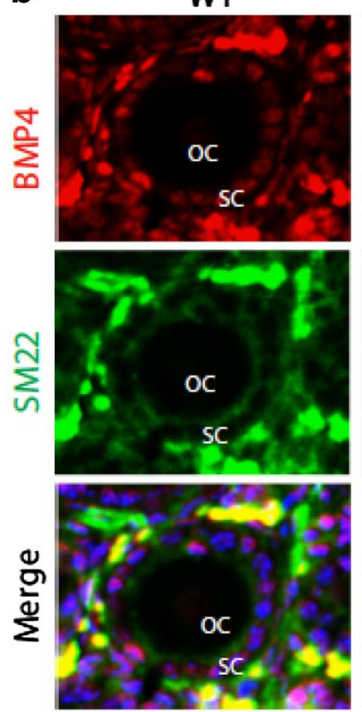

d
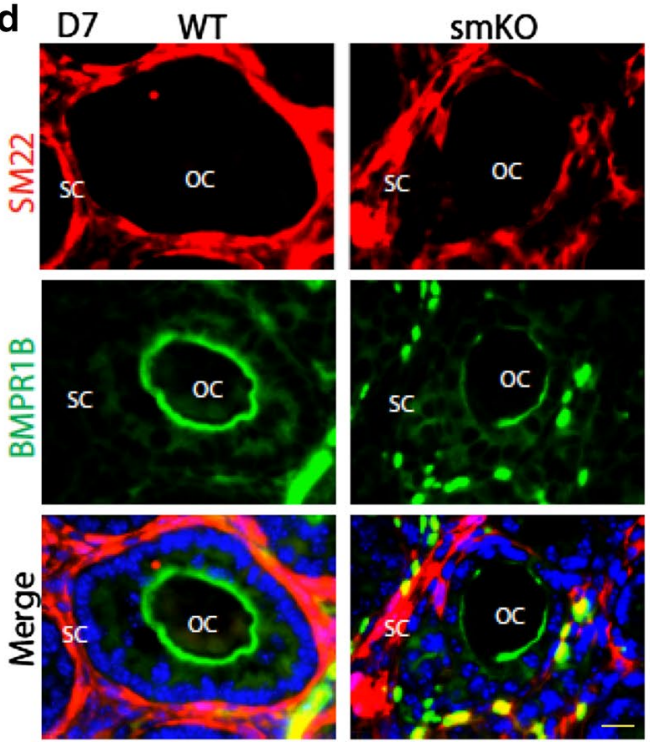

smKO
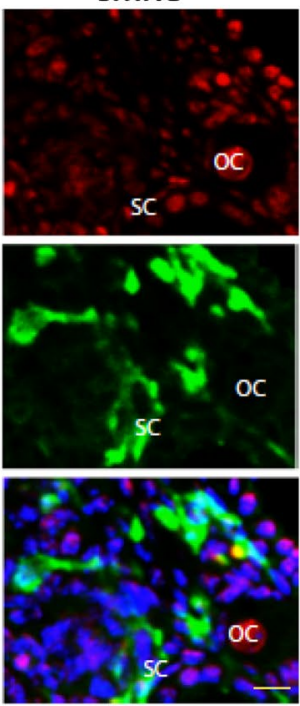

smKO
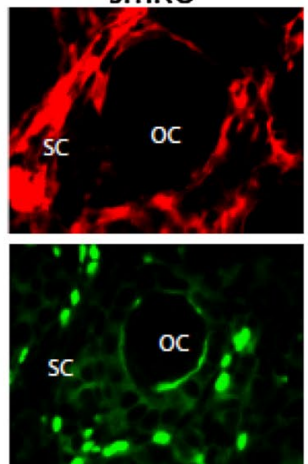
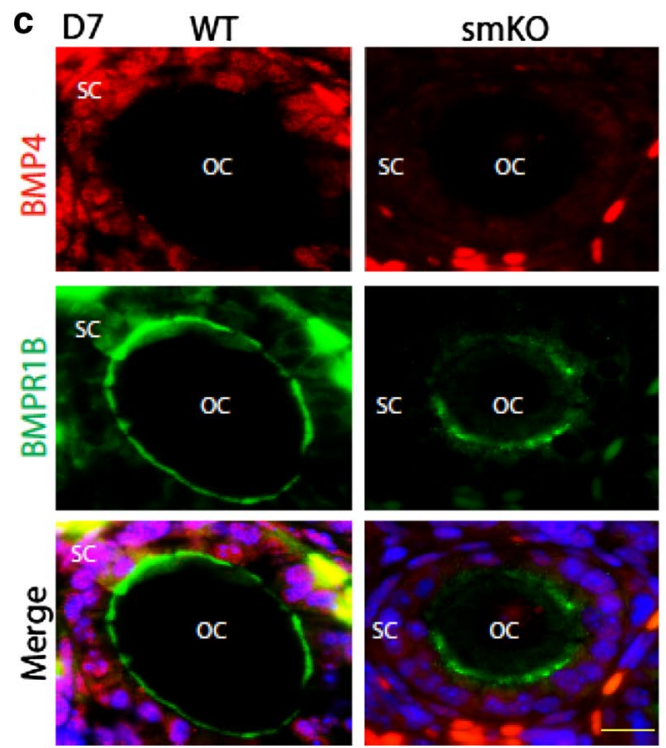

e
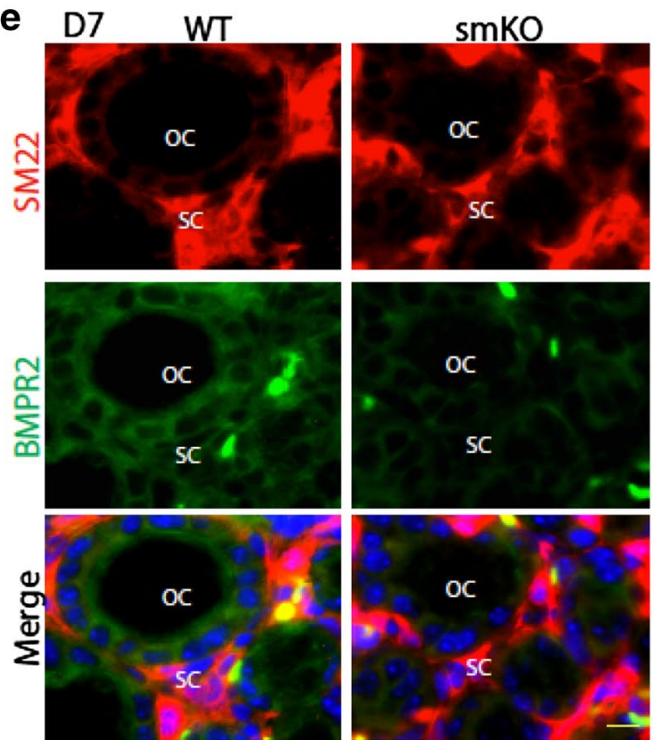

Fig. 5 BMPR1b expression in developing oocytes was reduced in SENP1-smKO mice. Ovaries from WT and SENP-smKO mice were harvested at postnatal day 7. a Protein levels of BMP4 and receptors were determined by western blot with respective antibodies. Normalized protein levels (with GADPH) are presented as fold changes by taking WT group as 1.0. $n=3$. b BMP4 and SM22a co-immunofluorescence staining of ovaries from WT and SENP-smKO mice. c BMP4 and BMPR2 co-immunofluorescence staining of ovaries from WT and SENP-smKO mice. d, e SM22a and BMPR1B or BMPR2 co-immunofluorescence staining of ovaries from WT and SENP-smKO mice at postnatal day 7. Scale bars $15 \mu \mathrm{m}$. OV ovary, OC oocyte, GC granulosa cell, SC stromal cell. Asterisks indicate the same oocyte in the merged panel 
In mammals, the growth of oocytes and development of follicles within the ovary are highly dependent on autocrine and paracrine growth factors from granulosa cells, theca cells, stromal interstitial cells, and the oocytes [27-32]. The bone morphogenetic protein (BMP) family belongs to the transforming growth factor (TGF- $\beta$ ) superfamily. BMPs bind to type I and type II serine-threonine kinase receptors, and transduce signals through the SMAD signaling pathway. Among BMP proteins, BMP4 and BMP-7 are reported to be of stromal cell and/ or pre-thecal origin [27-32]. BMP4 and its receptors BMPR-IB and BMPR-II (and BMPR-IA, weakly) have been detected in mouse ovaries [27, 28, 30-32]. Our data indicate that SENP1 deficiency in $\mathrm{SM} 22^{+}$mouse stromal cells, effectively reduces BMP4 mRNA and protein expression in ovary. Knockdown of SENP1 in SM22 ${ }^{+}$ human ovarian stromal cells also effectively attenuates expression of BMP4, but not BMP2 or BMP6. It has been experimentally confirmed that BMP4 promotes primordial follicle development and the primordial-to-primary follicle transition. Mice treated with BMP4 have significantly higher proportion of developing primary follicles and fewer arrested primordial follicles than untreated controls. Conversely, mice treated with neutralizing antibody against BMP4 have markedly smaller ovaries, associating with a progressive loss of oocytes and primordial follicles, accompanying loss of normal ovarian tissue morphology over time. More interestingly, BMP4 protein is localized in stromal cell populations associated with developing primordial follicles [29]. Taken together, SENP1, by regulating BMP4 expression in stromal cells concomitant with decreased expression of BMP4 receptor BMPR1b and BMPR2 on oocytes, promotes ovarian maturation (Fig. 6).

Regulation of BMP4 gene expression has been investigated non-ovarian stromal cells and results suggest that BMP4 expression is regulated by several transcriptional factors. Transcriptional factor SOX2 could negatively regulate $\mathrm{BMP} 4$ promoter activity, possibly through binding to the promoter located in the first intron region of BMP4. Interestingly, SOX2 can be SUMOylated at the lysine 247 and this modification inhibits the DNA binding of SOX2 [33]. SOX2 is a member of the high mobility group (HMG) domain DNA-binding proteins for transcriptional control and chromatin architecture. The HMG domain of SOX2 binds the DNA to facilitate transactivation by the cooperative transcription factors such as OCT3/4. Therefore, SOX2 together OCT3/4 regulate many critical genes involved in stem cell marker genes and developmental genes. Similar to SOX2, OCT3/4 are also regulated by SUMOylation [34]. The BMP4 gene promoter also contains an AP-1 element therefore BMP4

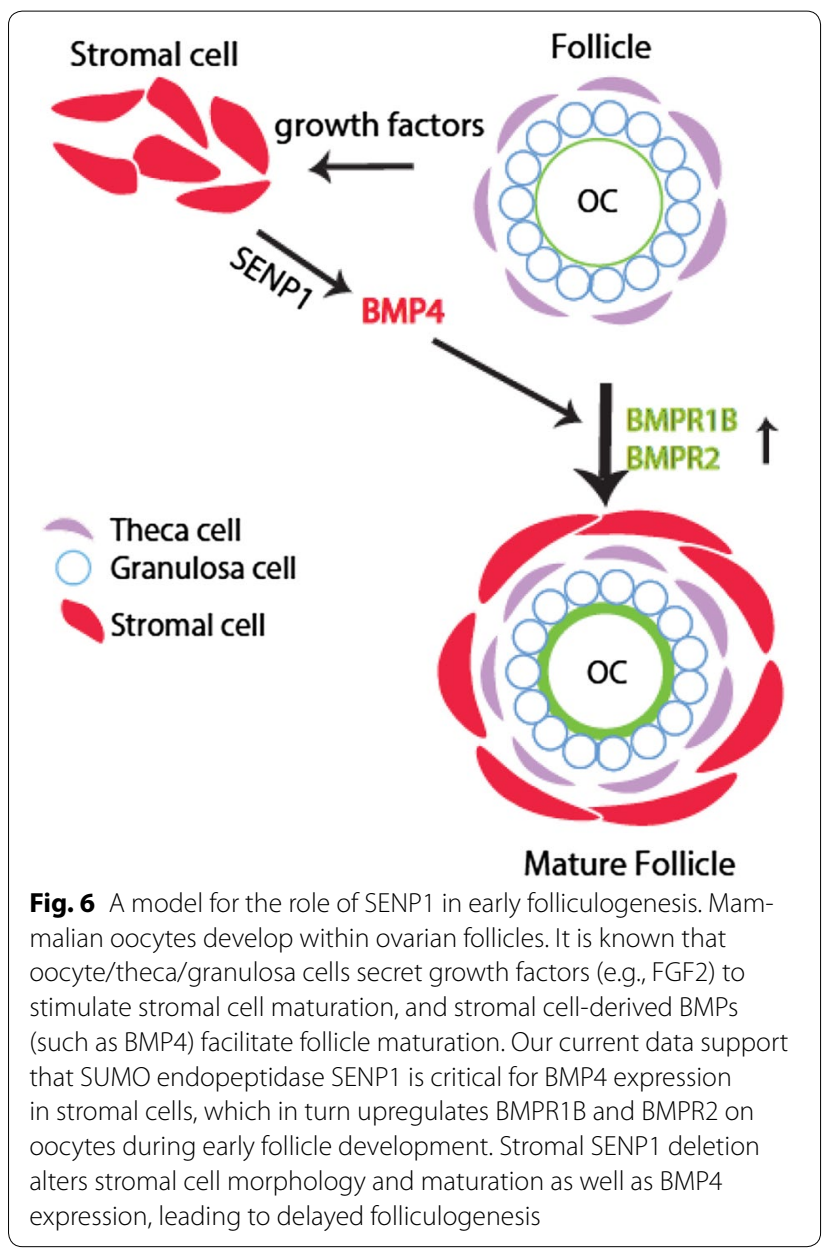

expression can be regulated by transcriptional factor $\mathrm{AP}-1$, and the integrin receptor, ILK, p38, and JNK signaling pathways [35]. It is well documented that AP-1 and upstream signaling are regulated by SUMOylation. We will investigate if SENP1, by modulating SUMOylation of SOX2-OCT1/4 or AP-1, regulate BMP4 expression in the ovarian stromal cells. BMP4 expression is also regulated at mRNA levels. LincRNA MEG3, via suppressing SOX2, positively regulates BMP4 transcription. Specifically, MEG3 could dissociate the transcription factor SOX2 from the BMP4 promoter [36]. Lin28, a stem cell factor, binds to BMP4 mRNA, thereby promoting BMP4 expression at the post-transcriptional level [37]. It has not been explored if LncRNA MEG3 and Lin28 are regulated by SUMOylation. Taken together, our study warrantee further investigation to define the mechanisms by which SENP1-SUMO mediates BMP4 gene expression, which will provide potential therapeutic targets for human POI and other ovarian associated diseases such as ovarian cancer. 


\section{Conclusions}

The mechanism by which stromal cells and derived factors regulate oocyte development remains unclear. Our present study has revealed that protein SUMOylation-regulating enzyme SENP1 plays a critical role in early ovarian follicle development by regulating gene expression of BMP4 in stroma and stroma-oocyte communication.

\section{Methods}

Smooth muscle 22a(SM22a) specific SENP1 knockout mice SENP1 $1^{+/ l o x}$ mice were generated by inserting loxP sites surrounding the SENP1 gene exons 5 and 6, based on homologous recombination 17. SENP1 lox/lox mice were obtained by intercrossing SENP1 ${ }^{+/ l o x}$ mice. SENP $1^{\text {lox } / \text { lox }}$ mice were mated with three different deleter lines carrying the Cre recombinase driven by the SM22 $\alpha$ (obtained from Jackson Laboratory). All mice had been subsequently backcrossed onto the C57BL/6 background for 46th generations. The deletion of SENP1 in uterine stromal cells of SENP1 ${ }^{\text {lox/lox}}$ : Cre was verified by quantitative PCR with reverse transcription using primers amplifying exons 5-6 $[19,38]$ and SENP1 ${ }^{+/+}$and specific Cre or SENP $1^{\text {lox/lox }}$ mice used as controls. Mice were cared for in accordance with National Institutes of Health guidelines, and all procedures. All animal studies were approved by the Institutional Animal Care and Use Committee of Yale University.

\section{Immunofluorescence staining}

Antibodies used for immunofluorescent staining. Confocal microscopy images were taken with a Zeiss-LSM 700 microscope and evaluated using the ZEN2010 software. For mean fluorescence intensity measurements, confocal microscopy images were analyzed with ImageJ. Slides were observed using a Zeiss Axiovert 200 fluorescence microscope (Carl Zeiss Microlmaging; Thornwood, $\mathrm{NY}$ ), and images were captured using Openlab3 software (Improvision, Lexington, MA). For tissue, $5 \mu \mathrm{m}$ serial sections cut from frozen, OCT-embedded tissues were fixed in $-20^{\circ} \mathrm{C}$ acetone for $10 \mathrm{~min}$, dried for $15 \mathrm{~min}$, followed by the same blocking/antibody protocol for cells as listed above.

\section{Cell culture and RNA interference for SENP1}

Human ovarian stromal cells (HOSC) were obtained from Department of Obstetrics and Gynecology, Yale School of Medicine and grown in DMEM media supplemented with $2 \mathrm{mM}$ glutamine and 15\% FBS. In view of the established characteristics of siRNA- targeting constructs, we designed three pairs of siRNA oligonucleotides for SENP1: siRNA 21696: 5-GGAAAUGGAGAAAGAAAUA dTdT-3; siRNA21512: 5-GGA
CCAGCUUUCGCUUUCU dTdT-3; siRNA21605: 5-GGACAUUUGGACCGA UCUU dTdT-3. Three siRNAs were obtained similar knockdown efficiency. The corresponding scramble siRNA oligonucleotide for siRNA21512: 5-GGA CCA GCA UAC GCU UUCU dTdT-3, with two nucleotide mutations (underlined), was synthesized from Ambion (Austin, TX, USA). For each transient transfection, siRNAs $(10 \mu \mathrm{M})$ and normalized plasmid $(10 \mathrm{mM})$ were transfected into cells by Oligofectamine (Life Technologies, Inc.; Invitrogen), according to the manufacturer's instructions (Invitrogen). Cells were cultured for 48 or $72 \mathrm{~h}$ before harvest.

\section{Quantitative PCR (qRT-PCR)}

Total RNA was extracted from human tissues using the RNeasy Plus Mini Kit (74134, Qiagen), and then converted into cDNAs using the High Capacity cDNA Reverse Transcription Kit (4368814, Applied Biosystems) following the manufacturer's instruction. Quantitative PCR was performed with a CFX-96 (Bio-Rad) using the RT2 SYBR Green (330500, SA Biosciences). All values were normalized with GAPDH abundance. Data were presented as the average of triplicates $\pm \mathrm{SD}$.

\section{Western blot}

Murine and human primary myometrium cells were directly lysed in Laemmli sample buffer (Bio-Rad) containing $\beta$-mercaptoethanol. Lysates were resolved on BioRad precast gradient gels (4-20\%) and transferred onto nitrocellulose membranes. After blocking (5\% non-fat dried milk in Tris buffered saline (TBS) with $0.1 \%$ Tween$20)$, membranes were probed with antibodies (1:200) for BMP4 (ab39973), BMPR1A (ab38560), BMPR1B (ab78417) and BMPR2 (ab106266), using anti-GAPDH (1:2000; Cell signaling) as a loading control, overnight at $4^{\circ}$.

\section{Statistical analysis}

The differences of results of oocytes counting, westernblot and qRT-PCR were analyzed by student $t$ test. Statistical analyses in this study were performed using SAS software (version 9.1.4, SAS Institute, Cary, NC). All statistical tests were two-tailed, and $P$ values less than 0.05 were considered statistically significant.

\section{Additional file}

Additional file 1: Figure S1. Endothelial cell-specific deletion of SENP1 has no effects on the size or number of developing follicles. (A, B) Hematoxylin and Eosin staining of ovaries from WT and SENP1-ecKO mice at postnatal day 3 and day 7 . Scale bars: $160 \mu \mathrm{m}$. Follicle size in ovaries of WT and SENP1-ecKO mice are quantified. Data are presented as means \pm SEM, $n=5$. (C) Number of total follicle in ovaries of WT and SENP1-smKO mice at ages of 3,6 weeks and 8 months were quantified based on $H \& E$ staining. Data are presented as means $\pm S E M, n=5$. 


\section{Authors' contributions}

$\mathrm{ST}, \mathrm{BF}, \mathrm{MY}, J \mathrm{HZ}, \mathrm{GL}, \mathrm{WJ}, \mathrm{YL}$, and WM conceived the study, designed experiments and wrote the manuscript; ST, BF, MY, JHZ performed experiments. All authors read and approved the final manuscript.

\section{Author details}

${ }^{1}$ Department of Pathology and the Vascular Biology and Therapeutics Program, Yale University School of Medicine, New Haven, CT 06519, USA. ${ }^{2}$ Center for Translational Medicine, The First Affiliated Hospital, Sun Yat-sen University, Guangzhou 510080, China. ${ }^{3}$ Department of Gynecology Oncology, The Tumor Affiliated Hospital of Harbin Medical University, Harbin, China. ${ }^{4}$ State Key Laboratory of Ophthalmology, Zhongshan Ophthalmic Center, Sun Yat-sen University, Guangzhou, China.

\section{Acknowledgements}

Not applicable.

\section{Competing interests}

The authors declare that they have no competing interests.

\section{Availability of data and materials}

SENP1-smKO mice will be available to the scientific community once the paper is published.

\section{Consent for publication}

Yes, we agree.

\section{Ethics approval and consent to participate}

No human tissues or human subjects were used in this study. All animal studies were approved by the Institutional Animal Care and Use Committee of Yale University.

\section{Funding}

This work was partly supported by National Key Research and Development Program of China (2016YFC1300600), National Natural Science Foundation of China (No. 91539110) and Scientific Grants of Guangdong (Nos. 2015B020225002 and 2015A050502018) to WM; NIH Grants R01 HL109420 and HL115148 to WM.

\section{Publisher's Note}

Springer Nature remains neutral with regard to jurisdictional claims in published maps and institutional affiliations.

Received: 23 February 2017 Accepted: 21 July 2017

Published online: 25 July 2017

\section{References}

1. Choi Y, Rajkovic A. Genetics of early mammalian folliculogenesis. Cell Mol Life Sci. 2006;63(5):579-90.

2. Odor DL, Blandau RJ. Ultrastructural studies on fetal and early postnatal mouse ovaries. I. Histogenesis and organogenesis. Am J Anat. 1969;124(2):163-86.

3. Rajkovic A, Pangas SA, Ballow D, Suzumori N, Matzuk MM. NOBOX deficiency disrupts early folliculogenesis and oocyte-specific gene expression. Science. 2004:305(5687):1157-9.

4. Jagarlamudi K, Rajkovic A. Oogenesis: transcriptional regulators and mouse models. Mol Cell Endocrinol. 2012;356(1-2):31-9.

5. Pangas SA, Choi Y, Ballow DJ, Zhao Y, Westphal H, Matzuk MM, Rajkovic A. Oogenesis requires germ cell-specific transcriptional regulators Sohlh1 and Lhx8. Proc Natl Acad Sci USA. 2006;103(21):8090-5.

6. Pangas SA, Rajkovic A. Transcriptional regulation of early oogenesis: in search of masters. Hum Reprod Update. 2006;12(1):65-76.

7. Li R, Albertini DF. The road to maturation: somatic cell interaction and self-organization of the mammalian oocyte. Nat Rev Mol Cell Biol. 2013;14(3):141-52.

8. McLaughlin EA, Mclver SC. Awakening the oocyte: controlling primordial follicle development. Reproduction. 2009;137(1):1-11.
9. Pepling ME, Spradling AC. Mouse ovarian germ cell cysts undergo programmed breakdown to form primordial follicles. Dev Biol. 2001:234(2):339-51.

10. Goswami D, Conway GS. Premature ovarian failure. Hum Reprod Update. 2005:11(4):391-410.

11. Kezele P, Nilsson E, Skinner MK. Cell-cell interactions in primordial follicle assembly and development. Front Biosci. 2002;7:d1990-6.

12. Senbon S, Hirao Y, Miyano T. Interactions between the oocyte and surrounding somatic cells in follicular development: lessons from in vitro culture. J Reprod Dev. 2003;49(4):259-69.

13. Zhang H, Liu K. Cellular and molecular regulation of the activation of mammalian primordial follicles: somatic cells initiate follicle activation in adulthood. Hum Reprod Update. 2015;21(6):779-86.

14. Zhang H, Risal S, Gorre N, Busayavalasa K, Li X, Shen Y, Bosbach B, Brannstrom M, Liu K. Somatic cells initiate primordial follicle activation and govern the development of dormant oocytes in mice. Curr Biol. 2014;24(21):2501-8

15. Kashimada K, Pelosi $E_{1}$ Chen $H$, Schlessinger D, Wilhelm D, Koopman P. FOXL2 and BMP2 act cooperatively to regulate follistatin gene expression during ovarian development. Endocrinology. 2011;152(1):272-80.

16. Gill G. SUMO and ubiquitin in the nucleus: different functions, similar mechanisms? Genes Dev. 2004;18(17):2046-59.

17. Pickart CM. Mechanisms underlying ubiquitination. Annu Rev Biochem. 2001;70:503-33.

18. Yeh ET. SUMOylation and De-SUMOylation: wrestling with life's processes. J Biol Chem. 2009;284(13):8223-7.

19. Yu L, Ji W, Zhang H, Renda MJ, He Y, Lin S, Cheng EC, Chen H, Krause DS, Min W. SENP1-mediated GATA1 deSUMOylation is critical for definitive erythropoiesis. J Exp Med. 2010;207(6):1183-95.

20. Cheng J, Kang X, Zhang S, Yeh ET. SUMO-specific protease 1 is essential for stabilization of HIF1alpha during hypoxia. Cell. 2007:131(3):584-95.

21. Rodriguez A, Pangas SA. Regulation of germ cell function by SUMOylation. Cell Tissue Res. 2016:363(1):47-55.

22. Vigodner M, Shrivastava V, Gutstein LE, Schneider J, Nieves E, Goldstein M, Feliciano M, Callaway M. Localization and identification of sumoylated proteins in human sperm: excessive sumoylation is a marker of defective spermatozoa. Hum Reprod. 2013;28(1):210-23.

23. Yuan YF, Zhai R, Liu XM, Khan HA, Zhen YH, Huo LJ. SUMO-1 plays crucia roles for spindle organization, chromosome congression, and chromosome segregation during mouse oocyte meiotic maturation. Mol Reprod Dev. 2014;81(8):712-24

24. Cubenas-Potts C, Goeres JD, Matunis MJ. SENP1 and SENP2 affect spatial and temporal control of sumoylation in mitosis. Mol Biol Cell. 2013:24(22):3483-95.

25. Ihara M, Stein P, Schultz RM. UBE2I (UBC9), a SUMO-conjugating enzyme, localizes to nuclear speckles and stimulates transcription in mouse oocytes. Biol Reprod. 2008;79(5):906-13.

26. He Y, Zhang H, Yu L, Gunel M, Boggon TJ, Chen H, Min W. Stabilization of VEGFR2 signaling by cerebral cavernous malformation 3 is critical for vascular development. Sci Signal. 2010;3(116):ra26.

27. Ding $X$, Zhang $X, M u$ Y, Li Y, Hao J. Effects of BMP4/SMAD signaling pathway on mouse primordial follicle growth and survival via up-regulation of Sohlh2 and c-kit. Mol Reprod Dev. 2013:80(1):70-8.

28. Lee WS, Yoon SJ, Yoon TK, Cha KY, Lee SH, Shimasaki S, Lee S, Lee KA. Effects of bone morphogenetic protein-7 (BMP-7) on primordial follicular growth in the mouse ovary. Mol Reprod Dev. 2004;69(2):159-63.

29. Nilsson EE, Skinner MK. Bone morphogenetic protein-4 acts as an ovarian follicle survival factor and promotes primordial follicle development. Biol Reprod. 2003;69(4):1265-72.

30. Rossi RO, Costa JJ, Silva AW, Saraiva MV, Van den Hurk R, Silva JR. The bone morphogenetic protein system and the regulation of ovarian follicle development in mammals. Zygote. 2016:24(1):1-17.

31. Tanwar PS, McFarlane JR. Dynamic expression of bone morphogenetic protein 4 in reproductive organs of female mice. Reproduction. 2011;142(4):573-9.

32. Yi SE, LaPolt PS, Yoon BS, Chen JY, Lu JK, Lyons KM. The type I BMP receptor BmprlB is essential for female reproductive function. Proc Natl Acad Sci USA. 2001:98(14):7994-9.

33. Tsuruzoe S, Ishihara K, Uchimura Y, Watanabe S, Sekita Y, Aoto T, Saitoh $H$, Yuasa Y, Niwa $H$, Kawasuji M, et al. Inhibition of DNA binding of 
Sox 2 by the SUMO conjugation. Biochem Biophys Res Commun. 2006;351(4):920-6.

34. Wu Y, Guo Z, Wu H, Wang X, Yang L, Shi X, Du J, Tang B, Li W, Yang L, et al. SUMOylation represses Nanog expression via modulating transcription factors Oct4 and Sox2. PLoS ONE. 2012;7(6):e39606.

35. Tan TW, Huang YL, Chang JT, Lin JJ, Fong YC, Kuo CC, Tsai CH, Chen YJ, Hsu $\mathrm{HC}$, Cho DY, et al. CCN3 increases BMP4 expression and bone mineralization in osteoblasts. J Cell Physiol. 2012;227(6):2531-41.

36. Zhuang W, Ge X, Yang S, Huang M, Zhuang W, Chen P, Zhang X, Fu J, Qu J, Li B. Upregulation of InCRNA MEG3 promotes osteogenic differentiation of mesenchymal stem cells from multiple myeloma patients by targeting BMP4 transcription. Stem Cells. 2015;33(6):1985-97.

37. Ma W, Ma J, Xu J, Qiao C, Branscum A, Cardenas A, Baron AT, Schwartz P, Maihle NJ, Huang Y. Lin28 regulates BMP4 and functions with Oct4 to affect ovarian tumor microenvironment. Cell Cycle. 2013;12(1):88-97.

38. Shao L, Zhou HJ, Zhang H, Qin L, Hwa J, Yun Z, Ji W, Min W. SENP1-mediated NEMO deSUMOylation in adipocytes limits inflammatory responses and type-1 diabetes progression. Nat Commun. 2015;6:8917.

\section{Submit your next manuscript to BioMed Central and we will help you at every step:}

- We accept pre-submission inquiries

- Our selector tool helps you to find the most relevant journal

- We provide round the clock customer support

- Convenient online submission

- Thorough peer review

- Inclusion in PubMed and all major indexing services

- Maximum visibility for your research

Submit your manuscript at www.biomedcentral.com/submit 